\title{
Overexpression of a Vesicle Trafficking Gene, OsRab7, Enhances Salt Tolerance in Rice
}

\author{
Xiaojue Peng, ${ }^{1}$ Xia Ding, ${ }^{1}$ Tianfang Chang, ${ }^{1}$ Zhoulong Wang, ${ }^{1}$ Rong Liu, ${ }^{1}$ Xin Zeng, \\ Yaohui Cai, ${ }^{2}$ and Youlin $\mathrm{Zhu}^{1}$ \\ ${ }^{1}$ Key Laboratory of Molecular Biology and Gene Engineering of Jiangxi Province, College of life science, Nanchang University, Nanchang \\ 330031, China \\ ${ }^{2}$ Jiangxi Super-rice Research and Development Center, Nanchang 330200, China
}

Correspondence should be addressed to Xiaojue Peng; xiaojuepeng@gmail.com

Received 3 November 2013; Accepted 24 December 2013; Published 12 February 2014

Academic Editors: L. R. Ceci and V. Niknam

Copyright (C) 2014 Xiaojue Peng et al. This is an open access article distributed under the Creative Commons Attribution License, which permits unrestricted use, distribution, and reproduction in any medium, provided the original work is properly cited.

High soils salinity is a main factor affecting agricultural production. Studying the function of salt-tolerance-related genes is essential to enhance crop tolerance to stress. Rab7 is a small GTP-binding protein that is distributed widely among eukaryotes. Endocytic trafficking mediated by Rab7 plays an important role in animal and yeast cells, but the current understanding of Rab7 in plants is still very limited. Herein, we isolated a vesicle trafficking gene, $O s R a b 7$, from rice. Transgenic rice over-expressing OsRab7 exhibited enhanced seedling growth and increased proline content under salt-treated conditions. Moreover, an increased number of vesicles was observed in the root tip of OsRab7 transgenic rice. The OsRab7 over-expression plants showed enhanced tolerance to salt stress, suggesting that vacuolar trafficking is important for salt tolerance in plants.

\section{Introduction}

High soil salinity is a major environmental stress inhibiting plant growth and development, and it results in severe yield loss in agricultural crops. Plants exhibit various responses to increase their capability to resist salt stress. Recent studies demonstrated that proteins involved in intracellular membrane trafficking in the secretory and endocytic pathways play an important role in plant defense against pathogens and stress adaptation [1].

Vesicle trafficking has been traditionally viewed as a constitutive housekeeping process, but recent reports have described the involvement of intracellular vesicle trafficking in the resistance to environmental stresses. In E. coli, mutations that cause increased vesiculation enhanced bacterial survival upon challenge with stressing agents or the accumulation of toxic misfolded proteins [2]. In yeast, vesicle trafficking between the cytosoll and plasma membrane was inhibited by oxidative stress [3]. In Arabidopsis thaliana, the transgenic plants in which the expression of an autophagy-related gene
AtATG18a was knocked down were more sensitive to salt and osmotic stress [4].

The Rab family is conserved from yeast to animals and has been implicated in intracellular vesicle trafficking and membrane organization [5]. In Arabidopsis, it is reported that the Rab7-related protein appears to localize to the vacuolar membrane and regulate the vesicle fusion with the vacuole [6]. In soybean, however, the presence of Rab7related protein was found on both endosomes and tonoplasts, indicating that $R a b 7$ multivesicular bodies are participated in endocytosis pathway. In addition, many plant $R a b 7$ genes were shown to be affected by environmental stressors, and transgenic experiments demonstrated that the overexpression of the AtRab7 and PgRab7 could increase stress tolerance in transgenic Arabidopsis thaliana and transgenic tobacco, respectively [6,7].

OsRab7 transcripts were found to accumulated slightly under salt stress in rice [8], but direct evidence showing that the overexpression of $O s R a b 7$ can increase tolerance to salt stress has not been reported. In this paper, we prepared 
transgenic rice plants that overexpressed $O s R a b 7$ and tested their tolerance to salt stress. The transgenic plants exhibited enhanced tolerance to salt stress and increased numerous distributed vesicles in the root tips.

\section{Materials and Methods}

2.1. Plant Materials and Growth Conditions. The japonica rice variety Zhonghua 11 was used in this study. $\mathrm{T}_{0}, \mathrm{~T}_{1}$, and $\mathrm{T}_{2}$ progeny transgenic plants were grown at $25^{\circ} \mathrm{C}$ under $16 \mathrm{~h}$ of daylight in a greenhouse. For the physiological analysis, the plants were grown under a controlled temperature of $23^{\circ} \mathrm{C}$ at $70 \%$ relative humidity.

2.2. Gene Cloning and Rice Transformation. Total RNA was isolated from rice seedlings with Trizol reagent (Invitrogen). Reverse transcription was performed using MMLV Reverse Transcriptase (Invitrogen) according to the manufacturer's directions. To obtain the full length sequence of OsRab7, a pair of primers was designed according to the OsRab7 cDNA sequence. The primer sequence was forward: 5'-ATGGCTTCGCGCCGC-3', Reverse: $5^{\prime}$ CTAGCAGCAGCCTGATGATCTTG- $3^{\prime}$. The OsRab7 cDNA was digested with BamHI and ligated to the modified binary vector plasmid pCU [9]. The resulting constructs were designated $p O s R a b 7$. The japonica rice variety zhonghua 11 was transformed using Agrobacterium tumefaciens EHA-105, following the published procedures published by Hiei et al. [10].

2.3. Semiquantitative RT-PCR and Real-Time RT-PCR Analysis. Total RNA was isolated and cDNA was synthesized from transgenic and wild-type rice as described above. The actin gene was used as an internal control for RTPCR analysis. General PCR was performed using the following program: an initial denaturation at $94^{\circ} \mathrm{C}$ for $3 \mathrm{~min}$, followed by 25 cycles of $94^{\circ} \mathrm{C}$ for $45 \mathrm{~s}, 60^{\circ} \mathrm{C}$ for $45 \mathrm{~s}$, and $72^{\circ} \mathrm{C}$ for $1 \mathrm{~min}$, and a final extension at $72^{\circ} \mathrm{C}$ for $10 \mathrm{~min}$. The RT-PCR experiments were repeated three times, and the PCR products were detected in a $1 \%$ agarose gel with $1 \times$ TAE and EtBr. Real-time PCR was performed with a Rotor-Gene 2000 real-time thermal cycling system (Corbett Research) using the SYBR Green Real-time PCR Master Mix (TOYOBO). The reaction for OsRab7 using specific primers (sense $5^{\prime}$-AGCCGTGTGGTCTCTGAG$3^{\prime}$; antisense $5^{\prime}$-AATGGGTCCTTGAGAGTCAC-3') was performed at $95^{\circ} \mathrm{C}$ for $10 \mathrm{~s}$ followed by 40 cycles of $95^{\circ} \mathrm{C}$ for $10 \mathrm{~s}, 55^{\circ} \mathrm{C}$ for $10 \mathrm{~s}$, and $72^{\circ} \mathrm{C}$ for $10 \mathrm{~s}$. The reaction for actin as internal standard using specific primers (sense $5^{\prime}$-AGCATGAAGATCAAGGTGGTC-3'; antisense $5^{\prime}$ GCCTTGGCAATCCACATC- $3^{\prime}$ ) was performed in the same as that for OsRab7.

2.4. Preparation of Total Protein Extract From Rice. Total protein was extracted from transgenic seedling and wild-type seedlings. The tissues were ground into a fine powder in liquid nitrogen, and then $400-500 \mathrm{mg}$ of tissue was extracted in $1 \mathrm{~mL}$ of extraction buffer (66 mM Tris-Cl, $\mathrm{pH} 6.8,2 \%$ SDS,
2\% v/v 2-mercaptoethanol) at room temperature followed by centrifugation for $20 \mathrm{~min}$ at $40000 \times \mathrm{g}$ to remove the insoluble fraction. The proteins were precipitated at $-20^{\circ} \mathrm{C}$ for $1 \mathrm{~h}$ by the addition of $1 \mathrm{~mL}$ of an $8: 1$ mixture of ice-cold acetone and trichloroacetic acid with $0.1 \% \mathrm{v} / \mathrm{v} 2$-mercaptoethanol. The supernatant was discarded after centrifugation at $18000 \times \mathrm{g}$ for $15 \mathrm{~min}$ at $4^{\circ} \mathrm{C}$, and the pellet was washed in $1 \mathrm{~mL}$ of ice-cold acetone. The remaining acetone was removed by air drying at room temperature. The resulting pellet was redissolved in the protein extraction solution.

2.5. Western Blotting Analysis. A polyclonal antibody against OsRab7 was obtained by the injection of a purified OsRab7 peptide into rabbits. Equal amounts of protein from independent transgenic rice and wild-type rice plants were separated on a $12 \%$ SDS-PAGE gel at $4^{\circ} \mathrm{C}$ and then transferred onto a PVDF transfer membrane at $80 \mathrm{~V}$ for $2 \mathrm{~h}$. The membrane was incubated in $5 \% \mathrm{w} / \mathrm{v}$ and nonfat milk, $0.05 \% \mathrm{v} / \mathrm{v}$ Tween20 , in phosphate buffered saline (PBS) for $1 \mathrm{~h}$, washed three times in PBST (PBS, 0.05\% Tween-20) for $10 \mathrm{~min}$ each, and incubated in a 1:1000 dilution of rabbit antiserum overnight at $4^{\circ} \mathrm{C}$. After four washes with PBST, the membrane was incubated with goat anti-rabbit IgG conjugated with alkaline phosphatase (AP) in PBST solution for $2 \mathrm{~h}$. After four $10 \mathrm{~min}$ washes in PBST, the signal was visualized by chemiluminescent detection (Pierce, Rockford, IL) according to the manufacturer's protocol.

2.6. Evaluation of Transgenic Rice Lines during Salt Stress. $\mathrm{T}_{2}$ generation seeds of OsRab7 homozygous plants were used to assess the relative salinity tolerance. Six-day-old transgenic and wild-type seedlings grown in 1/2 MS solid medium were transferred and placed with roots pointing downward into liquid medium supplemented with $200 \mathrm{mM} \mathrm{NaCl}$ for 10 days to induce salt stress. The Data from 10 plants each of the wild-type and T1 transgenic lines were collected for each experiment, and the mean values and standard deviations were calculated and compared in phenotypic changes, the treated plants were carefully investigated and photographed. Each treatment was repeated three times.

2.7. Determination of Proline Content. To test the defense response triggered by OsRab7 overexpression in transgenic rice, $\mathrm{T}_{2}$ homozygous plants (trans3, trans5), and wild-type plants were assayed for their proline content. Tow-weekold seedlings of both wild-type and transgenic rice were salt-treated $(250 \mathrm{mM})$ for $12 \mathrm{~h}$. The determination of the free proline content was performed according to Bates et al. [11]. The absorbance of chromophore containing toluene was recorded at $520 \mathrm{~nm}$ in a Shimadzu UV-1700 spectrophotomerter (Columbia, USA). L-proline (Sigma, USA) was used for the preparation of a standard curve.

2.8. Observation of Vesicle Fluorescence. Root tips from $\mathrm{T}_{2}$ homozygous plants and wild-type plant were, respectively, incubated with $5 \mu \mathrm{g} / \mathrm{mL}$ FM4-64 (Sigma) for $12 \mathrm{~min}$ on ice. After being washed twice, the root tips were examined 


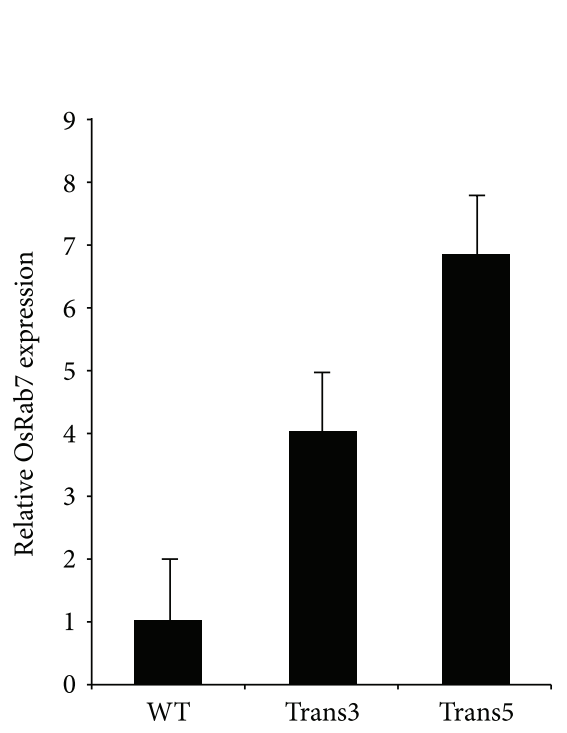

(a)

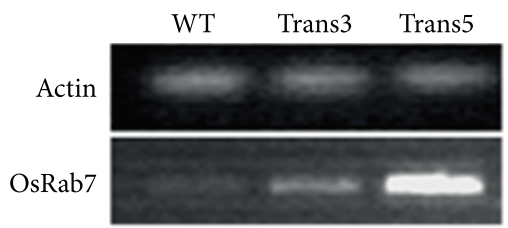

(b)

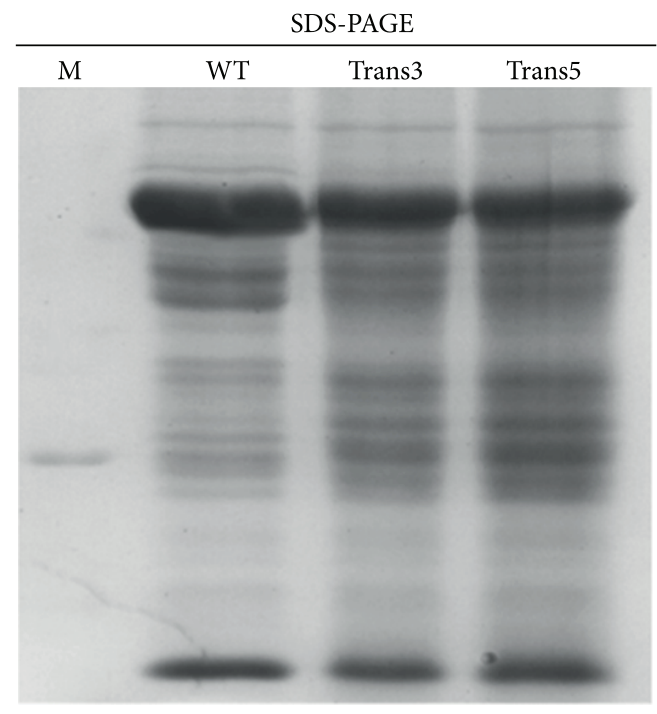

(c)

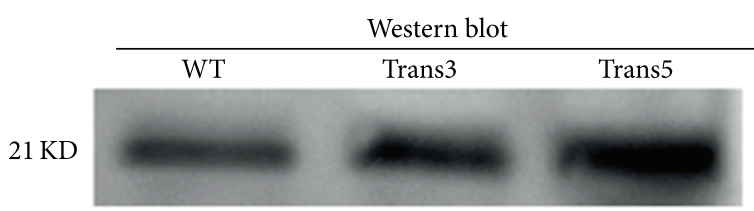

(d)

FIgURE 1: Expression of the OsRab7 gene in transgenic rice plants. (a) Real-time quantitative PCR analysis in transgenic plants. Total RNA was extracted from transgenic plant, and wild-type plants. Actin was used as an internal control. (b) OsRab7 expression in transgenic plants was determined by semiquantitative RT-PCR analysis. (c) a SDS-PAGE showed the protein content in the samples. Equal concentrations of protein extracts from the rice samples were separated by SDS-PAGE. (d) Western blot analysis of transgenic rice plants. All lanes were run with equal amounts of the protein extracts from the rice samples.

with a scanning confocal microscope (Olympus FV 1000). Excitation was $514 \mathrm{~nm}$ for the visualization of FM4-64.

\section{Results}

3.1. Molecular Characterization of Transgenic Rice Lines. To study the function of the OsRab7 gene in plants, the gene was cloned downstream of a constitutive ubiquitin promoter and introduced into rice plants via Agrobacterium tumefaciens-mediated transformation. Overall, 25 independent events were obtained and 23 transgenic events were confirmed by PCR firstly (Supplementary Material available online at http://dx.doi.org/10.1155/2014/483526). Analysis of the OsRab7 gene expression in the $\mathrm{T}_{2}$ progeny transgenic rice by semiquantitative RT-PCR and real-time RT-PCR showed that two homozygous lines (designated Trans 3 and Trans5) exhibited a high level of transgene overexpression. As shown in Figure 1(a), the transcript levels of OsRab7 increased to 4.2- and 7.8-fold, respectively, compared with the wild-type. Semiquantitative RT-PCR result also showed that the expression of OsRab7 increased in Trans 3 and Trans 5 (Figure 1(b)). Moreover, expression of the transgene at the protein level was assayed by western blot. Equal amounts of protein from transgenic and wild-type plant were separated by SDS-PAGE gel (Figure 1(c)). An increased amount of OsRab7 protein was obviously viable in Trans3 and Trans5 compared with the wild-type plants (Figure 1(d)).

3.2. Evaluation of OsRab7 Transgenic Rice Plants in Salt Stress. To analyze the impact of constitutive high OsRab7 expression on salt stress tolerance, the wild-type and the transgenic plants (Tans3 and Tran5) were subjected to salt stress by irrigation with $200 \mathrm{mM} \mathrm{NaCl}$. The treatment inhibited growth in both the wild-type and transgenic plants, but the transgenic plants were much less sensitive to the treatment compared with the wild-type plants (Figure 2(a)). The seedling growth of the transgenic plant and wild-type was investigated in more detail. The seedling length of Trans 5 was on average $0.18 \mathrm{~cm}, 0.22 \mathrm{~cm}$, and $0.81 \mathrm{~cm}$ longer than that of wild-type seedlings growing at 4, 7, and 10 days, respectively, in $200 \mathrm{mM}$ of $\mathrm{NaCl}$. We also measured the seedling length of Trans 3 at these stages and obtained the same results that the growth of OsRab7-overexpression transgenic rice was rapid compared with that of wild-type rice under salt stress (Figure 2(b)). Furthermore, the development of the lateral root in wild-type rice was delayed compared with that in 


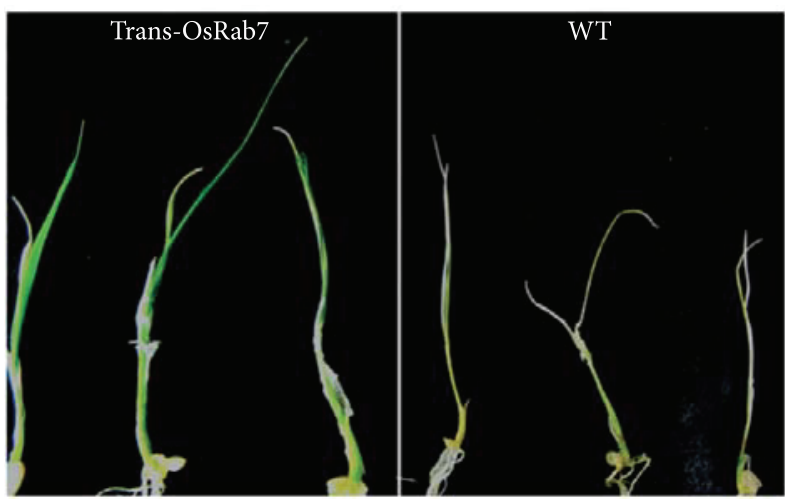

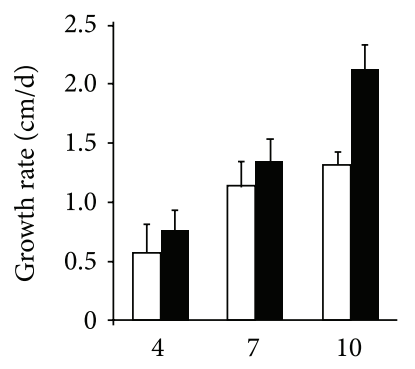

- WT

- Trans-OsRab7

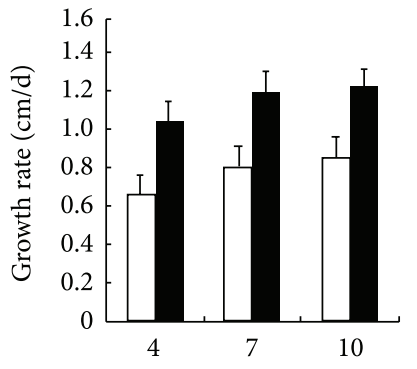

口 WT

- Trans-OsRab7

(a)

(b)

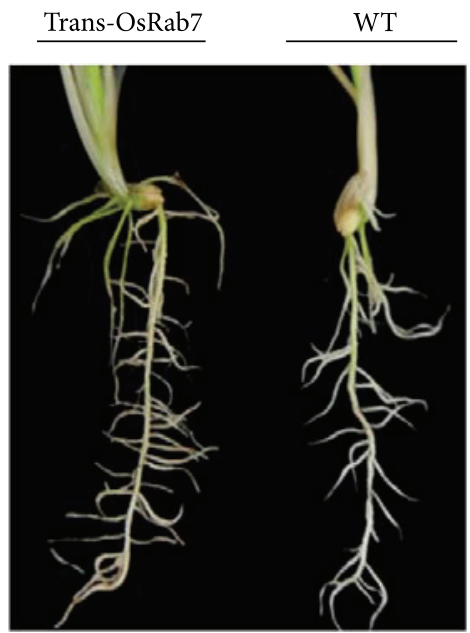

(c)

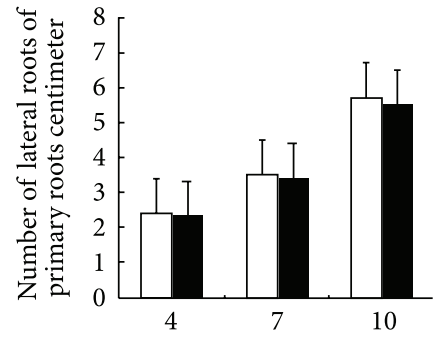

WT

- Trans-OsRab7

(d)

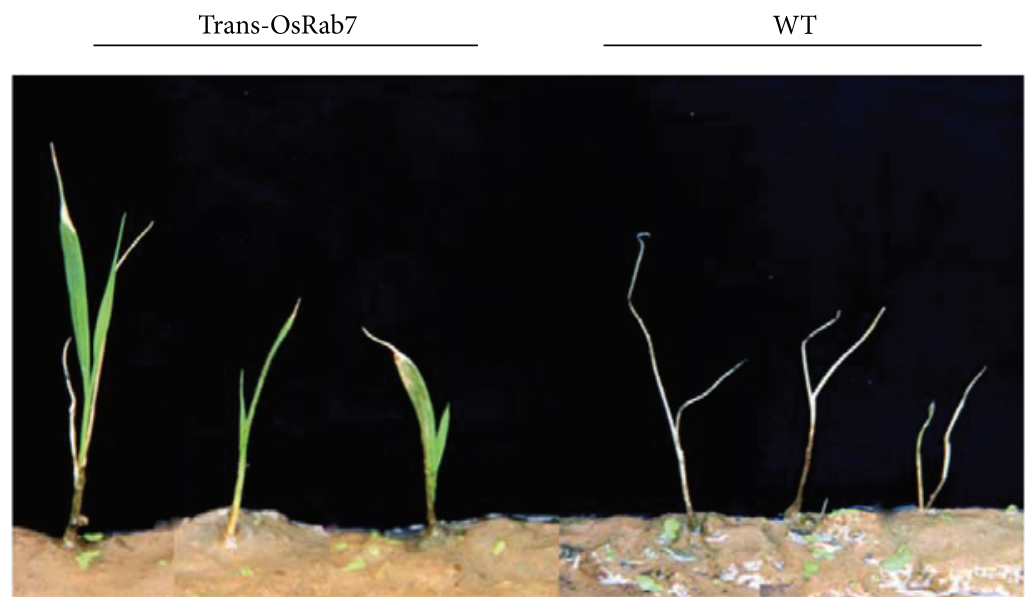

(e)

FIGURE 2: Salt stress tolerance in wild-type and transgenic plants. (a) Morphology of wild-type and transgenic seedlings after 12 days under $200 \mathrm{mM}$ salt stress. (b) Growth rate compared between the transgenic rice and WT plants under salt stress. (c) Root morphology under salt stress. (d) Analysis of the number of lateral roots at 4, 7, and 10 days. The data represents the mean \pm SD derived from three separate experiments. The bars represent the average data collected from 10 plants of each of the WT, Trans3, and Trans5 lines. (e) Morphology of wild-type and transgenic seedlings transplanted from salt liquid medium to soil. The wild-type and transgenic seedlings subjected to salt stress for 12 days were transplanted to soil, and the transgenic seedling growth was rescued after 8 days. 


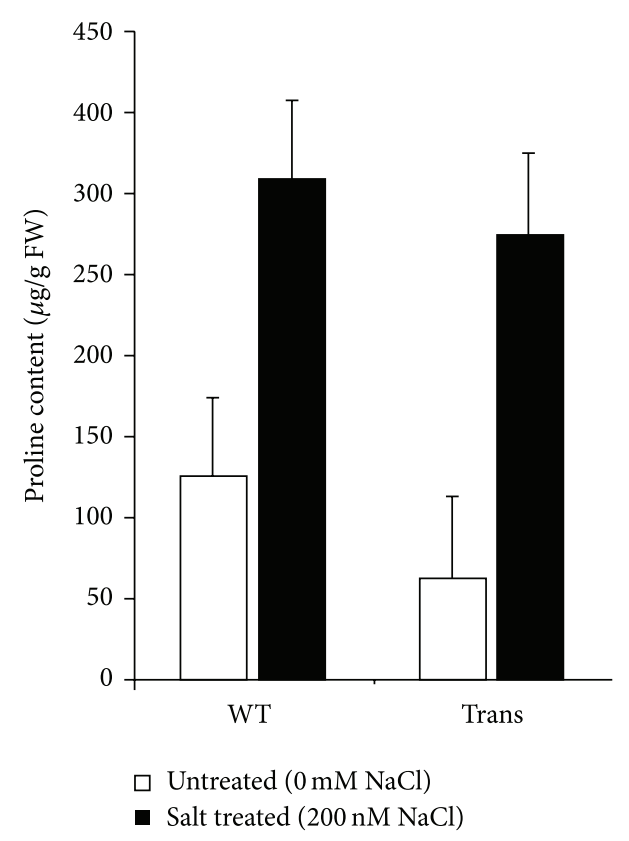

(a)

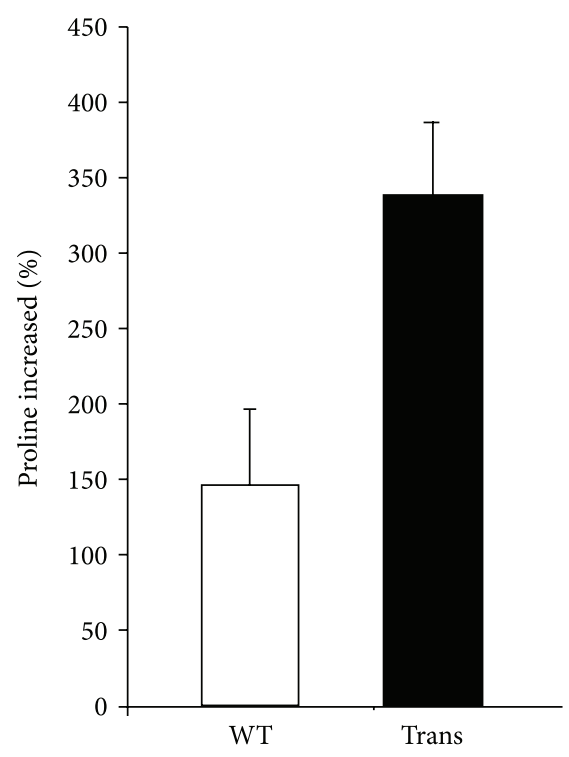

(b)

FIGURE 3: Overexpression of OsRab7 in rice increased the proline level under salt stress. (a) Level of proline in transgenic rice and WT rice under normal conditions and salt treatment. (b) Increasing rate of proline production after salt stress between the transgenic and WT rice. These data are the averages of triplicate assays and the error bars indicate the SDs.

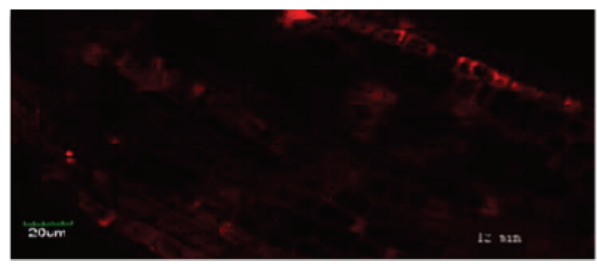

(a)

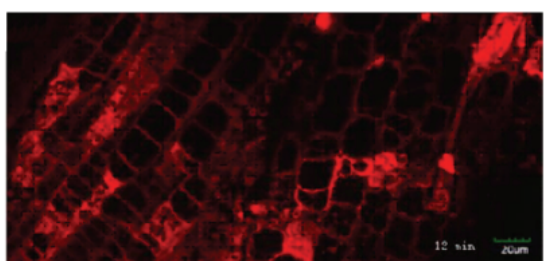

(b)

FIGURE 4: Vesicles in the transgenic plant and WT rice. (a) Vesicle morphology in the wild-type rice root tip. (b) Vesicle morphology in the transgenic plant root tip. The root tips were incubated in $5 \mu \mathrm{g} / \mathrm{mL}$ FM4-64 (Sigma) for $12 \mathrm{~min}$, and images of the vesicles were captured using confocal microscopy. Bar, $20 \mu \mathrm{m}$.

the transgenic plants under the salt stress (Figure 2(c)). The total number of lateral roots in the transgenic seedlings was higher than that of the wild-type seedling at 4,7 , and 10 days under salt stress. However, the number of lateral roots per centimeter was almost equal to that of the wildtype (Figure 4(d)). Almost all of the wild-type plants died 12 days after the beginning of the treatment with $200 \mathrm{mM}$ $\mathrm{NaCl}$, whereas many of the transformed plants still had green leaves (Figure 4(a)). To address the question of whether the seedling growth would resume after removing the salt stress, the wild-type and transgenic seedlings subjected to salt stress for 12 days were transplanted to soil. As shown in Figure 2(e), transgenic seedling growth was rescued after 8 days, but the wild-type seedling growth was not recovered at the same time (Figure 2(e)). Actually, transgenic rice plants produced seeds normally 4 weeks later, whereas the wild-type almost died (data not shown). These findings demonstrated that the overexpression of OsRab7 enhanced salt tolerance in rice.

\subsection{The Amount of Proline Increased in OsRab7 Trans-} genic Rice Plant in Salt Stress. Proline, a widely distributed osmolyte, plays an important role in enhancing abiotic stress tolerance. To investigate the physiological changes in the transgenic plants under salt stress, 2 -week-old seedlings were treated with $200 \mathrm{mM} \mathrm{NaCl}$ for $24 \mathrm{~h}$ and then used to determine the free proline content. Under normal conditions and salt-treated conditions, increased proline levels were found in both transgenic and wild-type plants, and the proline content in the transgenic rice being slightly lower than that in the wild-type (Figure 3(a)). However, upon salt treatment, the transgenic seedlings exhibited a 3.5-fold increased proline 
content level compared with those under normal conditions, whereas the proline content in the wild-type plants only increased 1.5-fold after the salt treatment (Figure 3(b)). Therefore, the results indicated that the overexpression of OsRab7 increased the proline content under salt-treated conditions, which helped transgenic seedlings to better adapt to the salt stress.

3.4. The Vesicles Increased in OsRab7 Transgenic Rice Plant. In plants, $R a b 7$ plays an important role in the vesicle trafficking process, and thus, the vesicle distribution in transgenic rice was investigated using the vesicle fluorescent dye FM4-64. As shown in Figure 4, a strong, aggregated fluorescence intensity of FM4-64 was observed in the transgenic rice root tips, whereas a much weaker, diffuse fluorescence intensity was detected in the wild-type root tip. This result suggested the acceleration of vesicles trafficking in the OsRab7 transgenic rice.

\section{Discussion}

The physiological response of plants to salt stress is a complex process requiring the coordinated function of many genes. Although OsRab7 transcript levels were to be found increased under salt stress, direct evidence for the association of this gene with salt tolerance was lacking. In this paper, our experiments showed that the overexpression of $O s R a b 7$ could confer salinity tolerance in transgenic rice.

Vacuoles play a critical role in salt tolerance by accumulating sodium, which is then removed from the cytosol, where it is toxic $[12,13]$. A connection between salt sensitivity and vacuolar trafficking has been well established in animal cells and yeast. Post-Golgi organelles involved in vacuolar trafficking were shown to be targets of salt stress, resulting in inefficient vacuolar trafficking in yeast and mammalian cells [14-16]. In plants, Leshem et al. reported that the endocytic pathway to the vacuole was also important for salt tolerance in Arabidopsis [17, 18]. The Rab7 proteins are important components of the vesicle trafficking system in all eukaryotes [5]. In animals and yeast, the Rab7 protein localizes to late endosomes and to lysosomes/vacuoles [19-22]. In plants, the Rab7 proteins have been localized to the tonoplast in both Arabidopsis and rice [8,23]. Moreover, enhanced vesicle trafficking and endocytic trafficking were also observed in Rab7 overexpression transgenic soybean and in animal cells containing constitutive active forms of Rab7 [24, 25], respectively. In our studies, an increased number of distributed vesicles was observed in OsRab7 overexpression transgenic rice, suggesting that the overexpression of OsRab7 may activate the vesicle trafficking in rice. Together with the results of salt tolerance displayed by the OsRab7 overexpression transgenic rice, we infer that the overexpression of OsRab7 increases plant salt tolerance via enhanced vesicle trafficking in rice. Thus, our results provide additional evidence that vacuolar trafficking is important for salt tolerance.

\section{Conclusion}

In summary, we prepared transgenic rice plants that overexpressed OsRab7 and tested their tolerance to salt stress. The transgenic plants exhibited enhanced tolerance to salt stress and increased numerous distributed vesicles in the root tips. These results indicate that the overexpression of OsRab7 increased plant salt tolerance via enhanced vesicle trafficking in rice.

\section{Conflict of Interests}

The authors declare that there is no conflict of interests.

\section{Authors' Contribution}

$\mathrm{X}$. Peng and X. Ding have contributed equally in this work.

\section{Acknowledgments}

This work was supported by the Natural Science Foundation of China (nos. 31160270, 31160019), the Youth Natural Science Foundation of Jiangxi (No. 20114BAB214009), the Project of Jiangxi Provincial Department of Education (no. GJJ13102), and the earmarked fund for Modern Agro-industry Technology Research System (no. CARS-01-04).

\section{References}

[1] C. J. Carter, S. Y. Bednarek, and N. V. Raikhel, "Membrane trafficking in plants: new discoveries and approaches," Current Opinion in Plant Biology, vol. 7, no. 6, pp. 701-707, 2004.

[2] A. J. McBroom and M. J. Kuehn, "Release of outer membrane vesicles by Gram-negative bacteria is a novel envelope stress response," Molecular Microbiology, vol. 63, no. 2, pp. 545-558, 2007.

[3] A. Levine, B. Belenghi, H. Damari-Weisler, and D. Granot, "Vesicle-associated membrane protein of arabidopsis suppresses bax-induced apoptosis in yeast downstream of oxidative burst," Journal of Biological Chemistry, vol. 276, no. 49, pp. 46284-46289, 2001.

[4] Y. Liu, Y. Xiong, and D. C. Bassham, "Autophagy is required for tolerance of drought and salt stress in plants," Autophagy, vol. 5, no. 7, pp. 954-963, 2009.

[5] M. Zerial and H. McBride, "Rab proteins as membrane organizers," Nature Reviews Molecular Cell Biology, vol. 2, no. 2, pp. 107-117, 2001.

[6] A. Mazel, Y. Leshem, B. S. Tiwari, and A. Levine, "Induction of salt and osmotic stress tolerance by overexpression of an intracellular vesicle trafficking protein AtRab7 (AtRabG3e), Plant Physiology, vol. 134, no. 1, pp. 118-128, 2004.

[7] P. K. Agarwal, P. Agarwal, P. Jain, B. Jha, M. K. Reddy, and S. K. Sopory, "Constitutive overexpression of a stress-inducible small GTP-binding protein PgRab7 from Pennisetum glaucum enhances abiotic stress tolerance in transgenic tobacco," Plant Cell Reports, vol. 27, no. 1, pp. 105-115, 2008.

[8] M. Y. Nahm, S. W. Kim, D. Yun, S. Y. Lee, M. J. Cho, and J. D. Bahk, "Molecular and biochemical analyses of OsRab7, a rice Rab7 homolog," Plant and Cell Physiology, vol. 44, no. 12, pp. 1341-1349, 2003. 
[9] R. Chen, X. Zhao, Z. Shao et al., "Rice UDP-glucose pyrophosphorylasel is essential for pollen callose deposition and its cosuppression results in a new type of thermosensitive genic male sterility," Plant Cell, vol. 19, no. 3, pp. 847-861, 2007.

[10] Y. Hiei, S. Ohta, T. Komari, and T. Kumashiro, "Efficient transformation of rice (Oryza sativa L.) mediated by Agrobacterium and sequence analysis of the boundaries of the T-DNA," Plant Journal, vol. 6, no. 2, pp. 271-282, 1994.

[11] L. S. Bates, R. P. Waldren, and I. D. Teare, "Rapid determination of free proline for water-stress studies," Plant and Soil, vol. 39, no. 1, pp. 205-207, 1973.

[12] M. P. Apse, G. S. Aharon, W. A. Snedden, and E. Blumwald, "Salt tolerance conferred by overexpression of a vacuolar $\mathrm{Na}+\mathrm{H}+$ antiport in Arabidopsis," Science, vol. 285, no. 5431, pp. 12561258, 1999.

[13] L. Xiong, H. Lee, M. Ishitani et al., "Repression of stress-responsive genes by FIERY2, a novel transcriptional regulator in Arabidopsis," Proceedings of the National Academy of Sciences of the United States of America, vol. 99, no. 16, pp. 10899-10904, 2002.

[14] C. Bachert, T. H. Lee, and A. D. Linstedt, "Lumenal endosomal and Golgi-retrieval determinants involved in $\mathrm{pH}$-sensitive targeting of an early Golgi protein," Molecular Biology of the Cell, vol. 12, no. 10, pp. 3152-3160, 2001.

[15] J. M. Pardo, B. Cubero, E. O. Leidi, and F. J. Quintero, "Alkali cation exchangers: roles in cellular homeostasis and stress tolerance," Journal of Experimental Botany, vol. 57, no. 5, pp. 1181-1199, 2006.

[16] A. Hernández, X. Jiang, B. Cubero et al., "Mutants of the arabidopsis thaliana cation/H+ antiporter AtNHX1 conferring increased salt tolerance in yeast: the endosome/prevacuolar compartment is a target for salt toxicity," Journal of Biological Chemistry, vol. 284, no. 21, pp. 14276-14285, 2009.

[17] Y. Leshem, N. Melamed-Book, O. Cagnac et al., "Suppression of Arabidopsis vesicle-SNARE expression inhibited fusion of $\mathrm{H}_{2} \mathrm{O}_{2}$-containing vesicles with tonoplast and increased salt tolerance," Proceedings of the National Academy of Sciences of the United States of America, vol. 103, no. 47, pp. 18008-18013, 2006.

[18] Y. Leshem, L. Seri, and A. Levine, "Induction of phosphatidylinositol 3-kinase-mediated endocytosis by salt stress leads to intracellular production of reactive oxygen species and salt tolerance," Plant Journal, vol. 51, no. 2, pp. 185-197, 2007.

[19] F. Schimmoller and H. Riezman, "Involvement of Ypt7p, a small GTPase, in traffic from late endosome to the vacuole in yeast," Journal of Cell Science, vol. 106, part 3, pp. 823-830, 1993.

[20] F. Bruckert, O. Laurent, and M. Satre, "Rab7, a multifaceted GTP-binding protein regulating access to degradative compartments in eukaryotic cells," Protoplasma, vol. 210, no. 3-4, pp. 108-116, 2000.

[21] C. Bucci, P. Thomsen, P. Nicoziani, J. McCarthy, and B. van Deurs, "Rab7: a key to lysosome biogenesis," Biology of the Cell, vol. 11, no. 2, pp. 467-480, 2000.

[22] H. R. Pelham, "Insights from yeast endosomes," Opinion in Cell Biology, vol. 14, no. 4, pp. 454-462, 2002.

[23] C. Saito, T. Ueda, H. Abe et al., "A complex and mobile structure forms a distinct subregion within the continuous vacuolar membrane in young cotyledons of Arabidopsis," Plant Journal, vol. 29, no. 3, pp. 245-255, 2002.

[24] E. Limpens, S. Lvanov, W. van Esse, G. Voets, E. Fedorova, and T. Bisseling, "Medicago $\mathrm{N}_{2}$-Fixing symbiosomes acquire the endocytic identity marker Rab7 but delay the acquisition of vacuolar identity," Plant Cell, vol. 21, no. 9, pp. 2811-2828, 2009.

[25] S. Meresse, J.-P. Gorvel, and P. Chavrier, "The rab7 GTPase resides on a vesicular compartment connected to lysosomes," Journal of Cell Science, vol. 108, no. 11, pp. 3349-3358, 1995. 

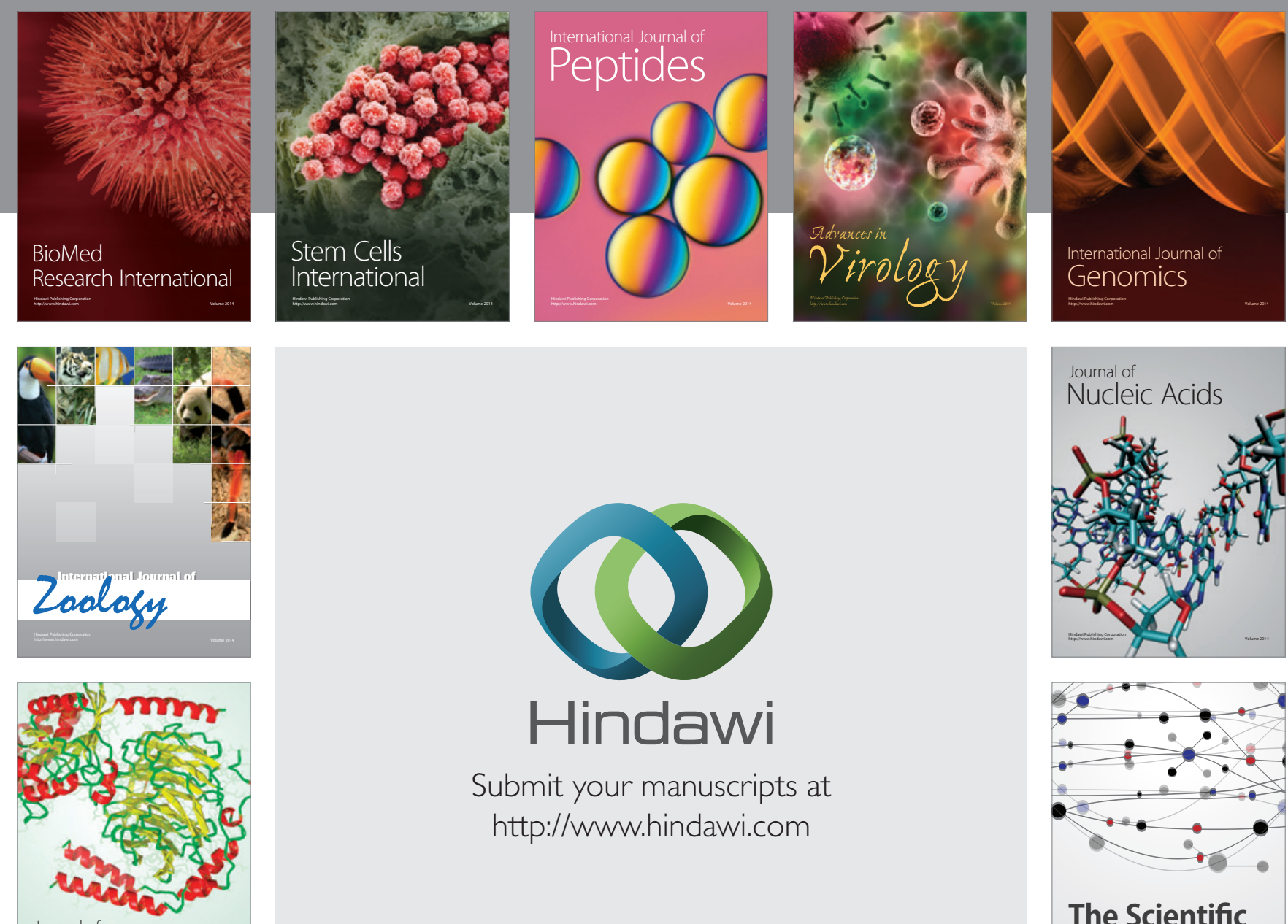

Submit your manuscripts at

http://www.hindawi.com

Journal of
Signal Transduction
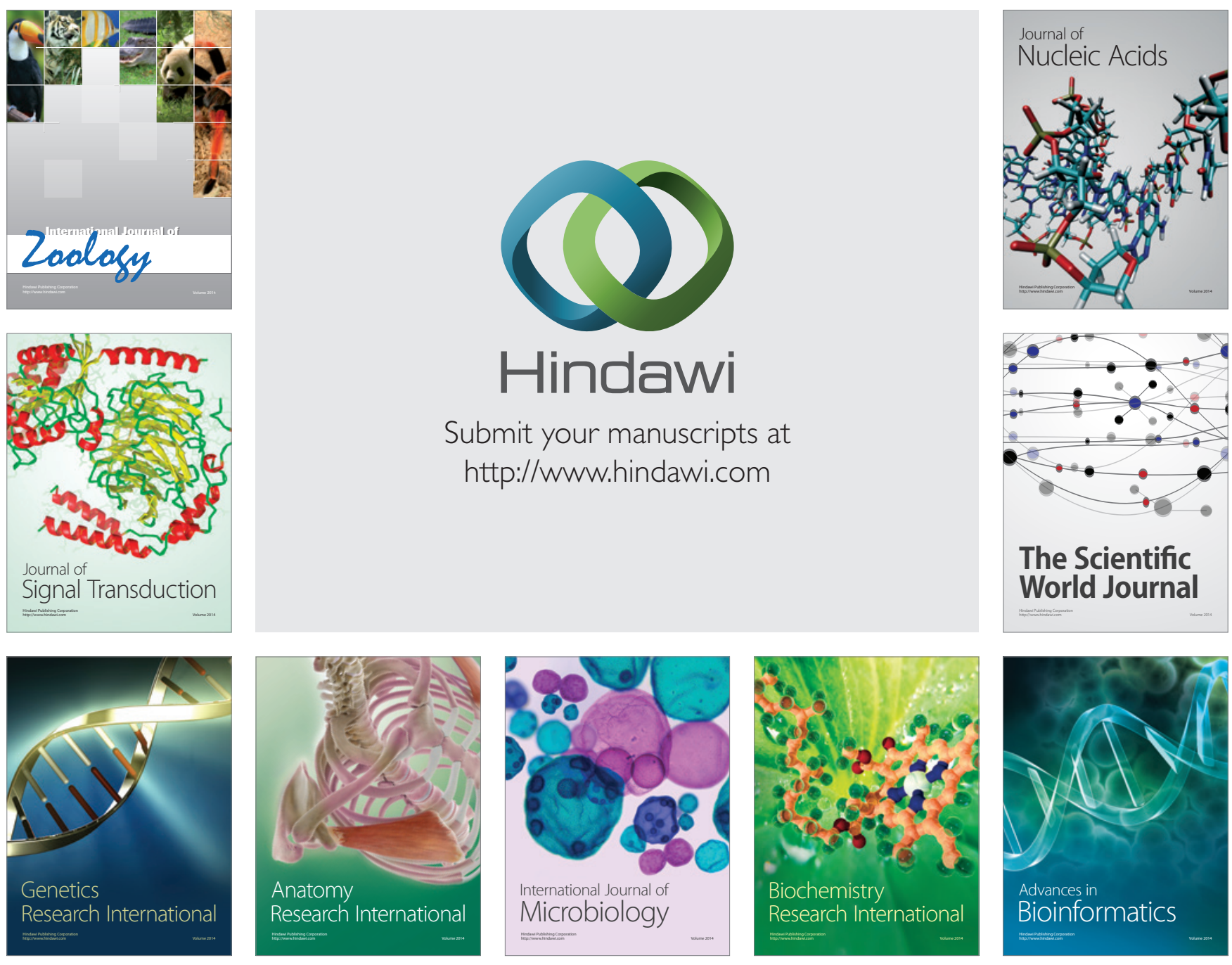

The Scientific World Journal
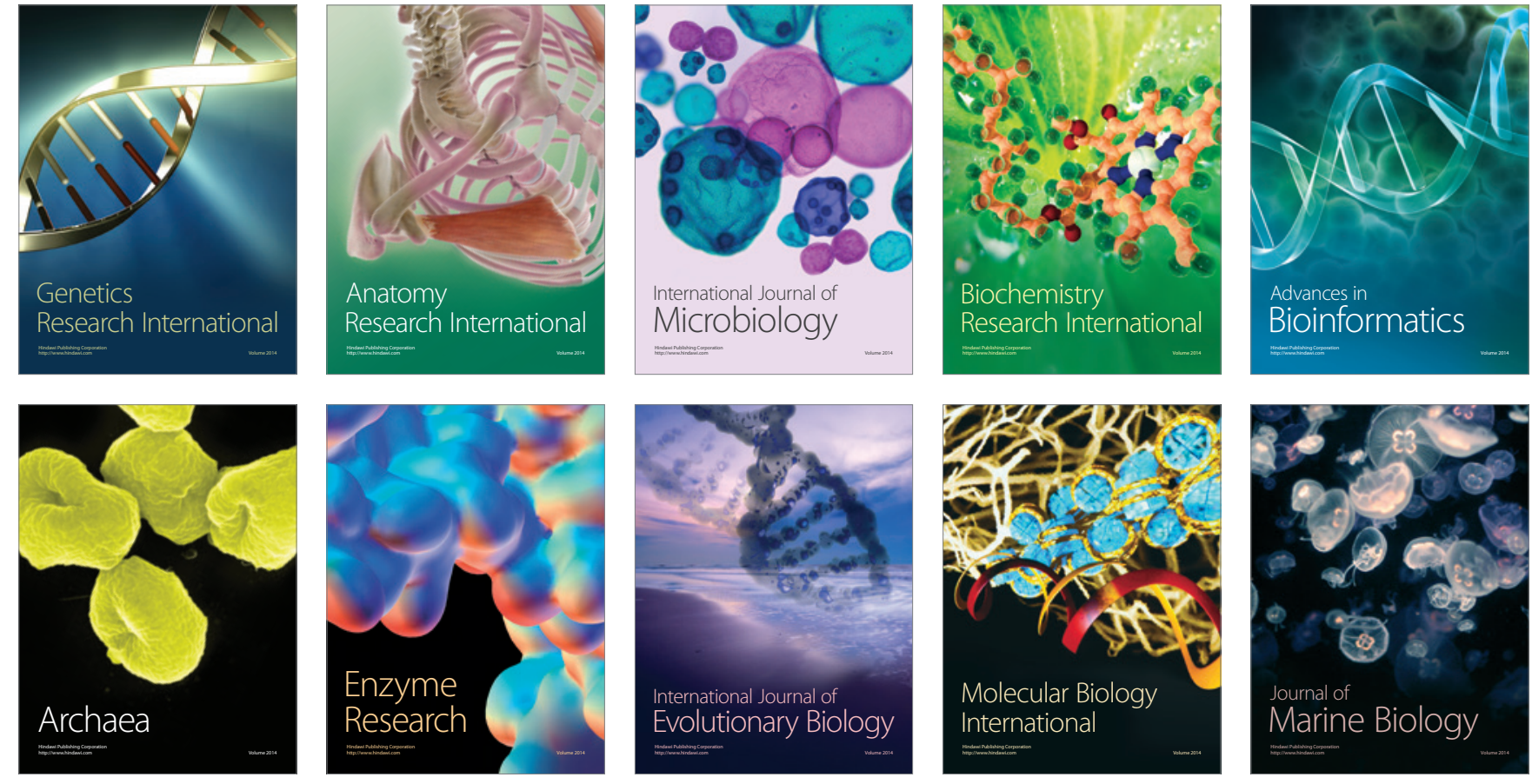